\title{
Effect of Pre-marital Sexual Counseling on Self-efficacy and Sexual Function of Iranian Newlywed Women
}

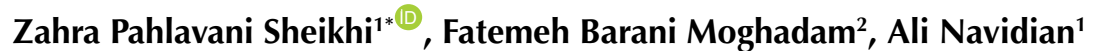

\begin{abstract}
Objectives: Sexual health counseling and education are important parts of pre-marital counseling programs, which can play effective roles in the prevention of sexual dysfunction and an increase in marital satisfaction. In this regard, this study aimed to assess the effect of the pre-marital sexual counseling of couples on self-efficacy and sexual function among females who referred to pre-marital training courses in Zahedan, Iran.

Materials and Methods: This quasi-experimental study was conducted on 110 couples before marriage, who were randomly divided into intervention and control groups. The intervention group was subjected to two 60-minute sexual counseling sessions whereas the control group received only the routine training in this regard. The obtained data were analyzed by SPSS software (version 21) using independent and dependent $t$ tests. A $P$ value of less than 0.05 was considered statistically significant.

Results: In this study, the mean score of sexual self-efficacy within the intervention group decreased from $20.61 \pm 6.05$ before attending the counseling sessions to $17.34 \pm 4.89$ three months after the intervention. However, the mean score of sexual self-efficacy within the control group represented a decrease from $17.87 \pm 5.49$ to $17.45 \pm 5.86$. Moreover, mean changes in the sexual self-efficacy scores of females in the intervention and control groups were $-3.27 \pm 1.16$ and $-0.42 \pm 0.37$, respectively. Furthermore, there was a significant difference between the intervention and control groups regarding the mean score of the sexual self-efficacy of females after the intervention $(P=0.01)$. In addition, there was a significant difference between the intervention and control groups in terms of the mean score of sexual function among females $(P=0.0001)$.

Conclusions: In summary, educational interventions and pre-marital counseling with regard to sexual health helped improve the marital relationships of couples.

Keywords: Sexual self-efficacy, Sexual function, Sexual counseling, Females, Pre-marital counseling
\end{abstract}

\section{Introduction}

Sexual function accompanied by a feeling of satisfaction is considered as one of the most fundamental dimensions of human life. As a result, healthy sexual function plays an important role in the health, happiness, and enhancement of the quality of life (1). It is estimated that more than half of the divorces are due to sexual dissatisfaction among Iranian couples (2-6). One of the factors affecting the sexual satisfaction of couples is the lack of information and insufficient knowledge regarding sexual and reproductive health (7-9).

Sexual self-efficacy is defined as an individual's belief in his or her capacity to be effective in sexual activities and be a pleasant sex partner (10). It is considered as a prerequisite for the change in the sexual behavior of individuals and is related to marital satisfaction (11-13).

Proper counseling and sexuality education are the key factors in the prevention of female sexual dysfunctions which can lead to the enhancement of women's sexual intelligence $(14,15)$. According to the literature, marital counseling and training should be provided at the nearest time to the marriage in order to have better effectiveness (16).

In many countries, there are pre-marital counseling programs for raising the levels of couples' knowledge regarding sexual health issues (17). After the approval of the National Plan in Iran in 1991, pre-marital counseling programs were held for all couples (18). Based on the studies on the quality of these programs, there was a need for reviewing and changing their structure $(18,19)$. The most important weaknesses of these training sessions include the insufficient content regarding sexuality education, the short duration of counseling sessions, the inadequacy of the educational environment, and teaching groups $(18,20)$.

With this background in mind, it is necessary to revise the pre-marital counseling programs currently held in Iran. Therefore, this study aimed to evaluate the effect of pre-marital sexual counseling on self-efficacy and sexual functions among women who referred to pre-marital training courses in Zahedan, Iran.

Received 4 February 2019, Accepted 9 July 2019, Available online 20 September 2019

'Pregnancy Health Research Center, Zahedan University of Medical Science, Zahedan, Iran. ${ }^{2}$ Nursing and Midwifery School, Zahedan University of Medical Science, Zahedan, Iran.

*Corresponding Author: Zahra Pahlavani Sheikhi, pahlavani_86@yahoo.com 
Key Messages

- The prevalence of sexual disorders among newlywed women throughout the world appears to be a major health problem.

- Sexual self-efficacy is an important prerequisite factor for changing sexual behaviors.

- Pre-marital counseling is one of the best opportunities for consultation and sexuality education which results in the improvement of sexual knowledge among the individuals

\section{Materials and Methods}

This study was performed with a pretest-posttest quasiexperimental design. The participants were randomly assigned to two intervention and control groups. In total, 110 participants were willing to participate in the study followed by a pre-test. They were all followed up during a 12 -week period and then evaluated after the completion of the post-test. The cases were asked to select randomly ordered envelopes, namely, A or B. The intervention and control groups included couples who chose envelope A and $\mathrm{B}$, respectively. Both groups were informed of the research objectives, and the control group was assured to obtain the training booklet at the end of the intervention.

\section{Population and Intervention}

The participants in this study were couples referring to the pre-marital counseling center for obtaining a marriage license in Zahedan. In addition, all subjects met the eligibility criteria. The inclusion criteria were the minimum age of 20 years, the first marriage of couples, reading and writing literacy, lack of receiving sex education before entering the study, and living with the spouse after marriage. Further, other criteria included the lack of special conditions leading to prevention from sex (e.g., recent stressful events such as diabetes, hypertension, and the like, lack of using certain medications including methyldopa and serotonin selective reuptake inhibitors, and finally, the absence of the previous history of surgery on the internal and external genitalia.

On the other hand, the exclusion criteria were females who experienced any stressful life events during the study (e.g., trauma, drug, alcohol addiction, and any incident or accident occurring in post-marital life), were unwilling to participate in sex educational sessions, and got divorced during the study.

These couples were all willing to participate in the study, completed demographic characteristics forms, and took the sexual self-efficacy pre-test before the receipt of the routine pre-marital counseling education. Both intervention and control groups were provided with routine sex education by related experts for females and males separately. Furthermore, the couples in the intervention group were given the training booklet and asked to study it before attending the next meeting, which will be held in the next two days. The contents of the training booklet included female and male genital anatomy, stages of natural sexual response, factors affecting desirable sexual function, and contraceptive methods and sexually transmitted diseases (STDs) in the next two days. The couples' contact numbers were also obtained for further coordination regarding participating in research sessions.

The major discussed topics in the first counseling session included an introduction to the female and male reproductive system, physiology of male sexual function, female's menstrual cycle, and contraceptive methods. The dimensions of sexual intercourse expectations, false beliefs and taboos, and different sex positions were highlighted in the second session (Table 1). These 60-minute sessions were held on two consecutive days. Individuals were divided into groups of five, and sessions were individually held for females and males with same-sex teachers. The participants in the control group received no additional education other than the routine training of the center. The sexual self-efficacy questionnaire was completed before the intervention by participants in both groups. Both intervention and control groups took and completed the post-test of sexual self-efficacy and the female sexual function questionnaires three months after the intervention. The researcher's contact number was given to the participants, and they were asked to come to the center for the post-test.

A questionnaire consisting of three parts was used in this study. The first part included demographic characteristics such as age, occupational status, level of education, ethnicity, and marital status. The second part of the questionnaire was a 19-item female sexual function index. This standard self-report questionnaire evaluates the sexual function of women regarding six independent subgroups of sexual desire (two items), sexual arousal (four items), sexual lubrication (four items), orgasm (three items), satisfaction (three items), and dyspareunia (three items). The total scores of the questionnaire were obtained by summing up the scores of the six subgroups, where the minimum and maximum scores were zero and 36 , respectively (21). The validity and reliability of this instrument were confirmed in Iran (22).

Table 1. The Content of Counseling Session

\begin{tabular}{ll}
\hline Session & Content \\
\hline Sexual counseling & $\begin{array}{l}\text { Introduction, female and male reproductive system, } \\
\text { physiology of male sexual function, female's } \\
\text { menstrual cycle, pregnancy, contraceptive methods, } \\
\text { and STDs }\end{array}$ \\
Sexual counseling & $\begin{array}{l}\text { The dimensions of sexual intercourse expectations, } \\
\text { session 2 }\end{array}$ \\
\hline
\end{tabular}

Note. STDs, sexually transmitted diseases. 
The third section was a sexual self-efficacy questionnaire designed by Vaziri and Lotfi Kashani in Iran based on Schwarzer's generalized self-efficacy scale. This 10-item questionnaire was scored based on a four-point Likerttype scale from zero (not true at all) to three (completely correct), and the range of the obtained scores varied from zero to 30 . In this questionnaire, higher scores indicated higher self-efficacy in individuals. The content validity of this self-efficacy questionnaire was confirmed in Iran. Moreover, its reliability was estimated at $0.86,0.81$, and 0.81 using Cronbach's alpha, the Spearman-Brown, and Guttman split-half reliability coefficient, respectively (10).

\section{Statistical Analysis}

Data analysis was performed by SPSS (version 21) using descriptive statistics (e.g., frequency distribution, mean, and standard deviation). With regard to qualitative data analysis, the chi-square test and the independent samples $\mathrm{t}$ test were applied to evaluate the mean of qualitative (i.e., education, occupational status, ethnicity, and habitation) and quantitative (age) variables, respectively. Finally, the paired $t$ test and covariance test were used to examine the effect of the intervention and control the pre-test effect.

\section{Results}

In total, 110 patients were enrolled in the study. The mean ages of females in the intervention and control groups were $24.67 \pm 3.94$ (with the age range of 20-35 years) and $26.36 \pm 4.01$ years (with the age range of $20-40$ years), respectively $(P=0.02)$. In addition, the mean duration of couples' pre-marital familiarity with each other in the intervention and control groups was $14.32 \pm 14.71$ (with a time interval of 1-60 months) and 9.62 \pm 8.49 (with a time interval of 1-30 months) months, which was significant according to the independent t-test $(\mathrm{P}=0.04)$.

In terms of occupational status, $52.73 \%$ and $58.18 \%$ of females in the intervention and control groups were housewives although the difference was not statistically significant $(P>0.05)$. Moreover, no significant differences were observed between the groups in terms of the level of education, ethnicity, independent lifestyle or living with parents, the way of getting acquainted with the spouse, marital satisfaction, and the sexual knowledge of females before the study $(P>0.05)$, the related data are provided in Table 2.

The results of this study showed that the mean score of self-efficacy in the intervention group changed from $20.60 \pm 61.05$ before the study to $17.34 \pm 4.89$ after the intervention. In the control group, the mean self-efficacy score of the subjects was $17.87 \pm 5.49$ at the beginning of the study, which changed to17.45 \pm 5.86 at the end of the research. Furthermore, the mean changes in terms of the self-efficacy scores of females in the intervention and control groups were $-3.1 \pm 27.16$ and $-0.42 \pm 0.37$, respectively. The results obtained from the independent $t$ test demonstrated a significant difference between the
Table 2. Demographic and Clinical Characteristics of Participants

\begin{tabular}{|c|c|c|c|}
\hline Characteristics & $\begin{array}{l}\text { Intervention } \\
\text { Group }(n=55)\end{array}$ & $\begin{array}{c}\text { Control Group } \\
(n=55)\end{array}$ & $P$ Value \\
\hline Age $($ Mean \pm SD) & $24.67 \pm 3.94$ & $26.36 \pm 4.01$ & 0.02 \\
\hline \multicolumn{4}{|l|}{ Level of education } \\
\hline Below diploma & $12(21.8)$ & $25(45.5)$ & \multirow{3}{*}{0.08} \\
\hline Diploma & $19(34.55)$ & $10(18.20)$ & \\
\hline Academic & $24(43.65)$ & $20(36.40)$ & \\
\hline \multicolumn{4}{|c|}{ Spousal education level } \\
\hline Below diploma & $20(46.5)$ & $15(27.2)$ & \multirow{3}{*}{0.17} \\
\hline Diploma & $24(44.6)$ & $20(36.3)$ & \\
\hline Academic & $11(0.2)$ & $20(36.3)$ & \\
\hline \multicolumn{4}{|c|}{ Occupational status } \\
\hline Housewife & $29(52.73)$ & $32(58.18)$ & \multirow[t]{2}{*}{0.78} \\
\hline Employed & $26(47.27)$ & $23(41.82)$ & \\
\hline \multicolumn{4}{|l|}{ Ethnicity } \\
\hline Sistani & $26(47.27)$ & $23(41.82)$ & \multirow{3}{*}{0.22} \\
\hline Baluch & $17(30.91)$ & $25(45.45)$ & \\
\hline Others & $12(21.82)$ & $7(12.73)$ & \\
\hline \multicolumn{4}{|l|}{ Habitation } \\
\hline Independent & $38(69.09)$ & $35(63.64)$ & \multirow[t]{2}{*}{0.54} \\
\hline With parent & $17(30.91)$ & $20(36.36)$ & \\
\hline \multicolumn{3}{|c|}{ Acquainted with the spouse } & \multirow{4}{*}{0.74} \\
\hline Traditional & $29(52.73)$ & $31(56.36)$ & \\
\hline Introduction & $18(37.73)$ & $16(29.09)$ & \\
\hline Others & $8(14.54)$ & $8(14.54$ & \\
\hline \multicolumn{4}{|l|}{ Marital satisfaction } \\
\hline Yes & $47(85.45)$ & $51(92.73)$ & \multirow[t]{2}{*}{0.22} \\
\hline No & $8(14.55)$ & $4(7.27)$ & \\
\hline \multicolumn{4}{|l|}{ Sexual knowledge } \\
\hline Yes & $12(21.82)$ & $8(14.55)$ & \multirow[t]{2}{*}{0.35} \\
\hline No & $43(78.18)$ & $47(85.45)$ & \\
\hline
\end{tabular}

intervention and control groups in terms of the mean selfefficacy scores of females after the intervention $(P=0.91)$. Based on the results (Table 3), the mean changes in selfefficacy scores were significant between the two groups $(P=0.006)$. The obtained results (Table 4) from the covariance analysis test with the control of the pretesting effect indicated a significant difference between the groups regarding the mean self-efficacy score $(P=0.0001)$.

The mean sexual function scores of the participants in the intervention and control groups were $23.61 \pm 4.50$ and $20.76 \pm 4.09$, respectively, three months after the intervention. Based on the obtained results (Table 5) from the independent t-test, a significant difference was found between the groups in terms of the mean score of sexual function after the intervention $(P=0.0001)$. Moreover, there was a significant difference between the mean scores of the intervention and control groups before and after the intervention regarding sexual desire and arousal, lubrication, satisfaction, and dyspareunia. However, no significant difference was observed between the groups in terms of orgasm $(P=0.32)$. 
Table 3. Comparison of the Mean of Self-efficacy Score in the Intervention and Control Groups Before and After the Intervention

\begin{tabular}{lccc}
\hline Self-efficacy Score & Experimental Group, Mean (SD) & Control Group, Mean (SD) $^{\boldsymbol{P}_{\text {Value }}}$ & 0.01 \\
\hline Pre-test score & $20.61 \pm 6.05$ & $17.87 \pm 5.49$ & $17.45 \pm 5.86$ \\
Post-test score & $17.34 \pm 4.89$ & $-0.42 \pm 0.37$ \\
Score gain & $-3.27 \pm 1.16$ & 0.91 \\
$P$ value & 0.01 & 0.90 \\
\hline
\end{tabular}

Note. SD, standard deviation.

andependent $t$ test.

Table 4. Covariance Analysis Related to the Self-efficacy Score in the Intervention and Control Groups

\begin{tabular}{|c|c|c|c|c|c|c|c|}
\hline Source of Change & Squares & Degrees of Freedom & Mean Squares & $\mathbf{F}$ & $P$ & Impact & Power \\
\hline Pretest & 930.39 & 1 & 930.39 & 44.89 & 0.0001 & 0.29 & 1 \\
\hline Group & 58.80 & 1 & 58.80 & 2.83 & 0.0001 & 0.02 & 1 \\
\hline Error rate & 2217.67 & 107 & 20.72 & & & & \\
\hline Total & 36452 & 110 & & & & & \\
\hline
\end{tabular}

Table 5. Distribution of FSFI Scores in the Intervention and Control Groups After the Intervention (N=110)

\begin{tabular}{lccc}
\hline Dimensions & Intervention Group, Mean (SD) & Control Group, Mean (SD) & $\boldsymbol{P}$ Value \\
\hline Sexual desire & $3.80 \pm 0.96$ & $3.38 \pm 0.94$ & 0.02 \\
Sexual arousal & $3.69 \pm 0.96$ & $3.08 \pm 0.9$ & 0.001 \\
Lubrication & $4.18 \pm 0.92$ & $3.63 \pm 0.74$ & 0.001 \\
Orgasm & $3.46 \pm 1.27$ & $3.25 \pm 0.82$ & 0.32 \\
Sexual satisfaction & $4.26 \pm 1.07$ & $3.62 \pm 0.90$ & 0.001 \\
Dyspareunia & $4.19 \pm 0.90$ & $3.78 \pm 0.77$ & 0.01 \\
\hline
\end{tabular}

Note. FSFI, female sexual function index; SD: standard deviation.

${ }^{a}$ Independent $t$ test.

\section{Discussion}

This study sought to evaluate the effect of pre-marital sexual health education on the self-efficacy and sexual function of females in Southeastern Iran. The obtained results indicated that sexual counseling had a positive effect on the sexual performance of these couples in the intervention group. In this regard, our findings are in line with the results of Alimohammadi et al who evaluated the effect of a group counseling kind's based on Bandura's self-efficacy theory on the sexual performance of newly married women in Zanjan, Iran. The results of the mentioned study revealed that the participants in the intervention group had a better sexual function after receiving sexual counseling compared to the control group (23).

Behboodi Moghadam et al conducted a study on 90 married females with sexual dysfunction attending a sexual health education program in Qazvin, Iran. They obtained similar results demonstrating an increase in the total sexual function scores of the intervention group after sexual training classes (24). According to the literature, counseling and sexual skill training sessions could be associated with better sexual performance and higher satisfaction of individuals $(2,25)$.

In another study performed in Tehran, pre-marital sexual counseling significantly increased sexual and marital satisfaction among 64 couples four months after the intervention (15). In addition, Parhizgar et al found that effective communication skills, problem solving, and proper sexual intercourse training could increase the marital satisfaction of 60 couples just before their marriage (16). In another study, Vural and Temel evaluated the effect of a pre-marital sexual counseling program on the sexual satisfaction of 71 Turkish couples. They also obtained similar results and concluded that those receiving this type of pre-marital education had higher levels of satisfaction (26).

Previous studies demonstrated that the quality of sexual life was directly correlated with satisfaction with the quality of relationships and public life. The desirable quality of sexual life results in more positive experiences in terms of sexual feelings leading to happiness and satisfaction with life (27-29). The role of sexual quality in marital satisfaction is one of the important factors in achieving happiness and satisfaction with life (1). Sexual intercourse with satisfaction is one of the most important pleasures that human beings gain throughout their lives. Consequently, this pleasure leads to the negligence of many marital conflicts and everyday life problems (30).

The prevalence of sexual disorders among women 
throughout the world appears to be a major health problem (31). In a systematic review, the prevalence of sexual dysfunctions among women of reproductive age was reported as $41 \%$ (32) although it was obtained $60 \%$ in Iran (11). According to the results from the subgroups of female sexual function, there was a significant difference between the intervention and control groups in terms of sexual desire, arousal, lubrication, sexual satisfaction, and dyspareunia. However, no significant difference was found between the two groups regarding orgasm. Women may not have orgasms due to various reasons such as anxiety, unfavorable mental conditions, and inaccurate sexual intercourse $(26,33,34)$. This difference in women's sexual function may be due to various causes including the culture and traditions governing the Iranian society. Many women consider sexual issues as taboos and are severely afraid of the first intercourse (26). Moreover, the issue of virginity on the wedding night worsens the situation as well (14).

Improper sexual intercourse might also occur as a result of a lack of foreplay. According to the results of the present study, although there was a significant difference in the mean self-efficacy score of women in the intervention group before and after the intervention, it was an adverse difference, and the women's self-efficacy scores represented a decline. Self-efficacy is a variable affecting the quality of sexual life. In addition, sexual self-efficacy is a belief one has about his/her own ability regarding effective sexual activities and being a desirable sex partner. Such a belief is considered a self-assessment of the ability and effectiveness of sexual behavior $(10,13)$. However, like many other countries, the discussions of sexual topics are generally regarded as a taboo among Iranians (35).

Premarital sex is not allowed in Iran due to the religious teachings of Islam. Therefore, the majority of Iranian Muslims require pre-marital consultation and sex education, especially the women whose lack of prior sexual experience reduces their self-esteem. Studies conducted in Iran have demonstrated that Iranian adolescents' have extremely inadequate knowledge of sexual health $(8,35)$. Due to cultural issues, sexuality education training is not provided in Iranian schools, and the majority of adolescents do not access proper information resources regarding gaining knowledge about sexual health. Friend groups are the only available sources and a great number of parents do not have a positive attitude toward providing their child with sexual education. Moreover, issues regarding sexual health are neglected by school instructors $(23,35,36)$.

According to the results from several studies, convincing people through discussions is a factor that affects selfefficacy. Educational group interventions can increase the knowledge of individuals and create a situation where people feel secure and overcome their fears (36). It has been reported that direct discussions about sexual issues are usually associated with shame in most societies
$(26,35)$. On the other hand, literature reviews suggest that individuals with higher education levels are more likely to talk about sexual issues (26). In the present study, subjects in the intervention group had higher education levels compared to the control group. Therefore, this variable may be one of the reasons for the change in the selfefficacy scores of the individuals.

It is assumed that self-efficacy is an important prerequisite factor for changing health behaviors. According to the literature, sexual self-efficacy can play an essential role in establishing proper sexual intercourse and can prevent sexual dysfunctions among people (13, 23). Perceived self-efficacy and basic skills are important factors for successful performance and behaviors changes, respectively (23). These results are in line with the findings of Vaziri et al on the relationship between sexual selfefficacy and marital satisfaction. They showed that sexual performance scores predicted marital satisfaction (10). In another study, Reissing et al concluded that increased sexual self-efficacy could be a solution for dealing with sexual problems among females (30). Furthermore, Zimmer-Gembeck conducted a study on 199 women aged 16-25 years and found that individuals with higher sexual self-efficacy had a better relationship with their sex partner (37). In this regard, Dillon and Beechler reported that the use of knowledge-raising programs for enriching sexual relations would increase satisfaction with marital life (36). Thus, pre-marital counseling is one of the best opportunities for consultation and sexuality education which results in the improvement of sexual knowledge among the individuals (38).

One of the strengths of this study was to provide training sessions for females and males. In this regard, an increase in males' knowledge can play a significant role in establishing better relations with their sexual partners leading to better sexual performance. Nonetheless, these courses were separately held for men and women due to cultural traditions governing society. Accordingly, it is suggested that future studies evaluate the impact of sexuality education programs on male sexual functions.

\section{Limitations}

One of the major limitations of the present study was the lack of knowledge regarding pre-marital sexual relations among individuals. Although the participants claimed to have the proper knowledge of sexual health, some cultural and religious barriers may deter them from telling the truth. This study was carried out on people living in Southeastern Iran. Therefore, the results may not be generalizable to other populations residing in other parts of the country.

\section{Conclusions}

According to the results of the present study, immediate post-marital initiation of regular sexual intercourse lead to lower levels of sexual self-efficacy in women. On the 
other hand, the correct learning of sexual issues leads to the prevention of sexual dysfunctions. Therefore, sexual health counseling and education are provided to pre-marital couples who can positively affect their sexual function. This lack of consistency might be due to an increase in the awareness and sensitivity of people, leading to a decrease in self-confidence and a belief that they cannot establish sexual relations as trained. Based on the results of this investigation, it seems that pre-marital interventions with regard to sexual health help improve the marital relationship of individuals. In other words, it could be stated that counseling and education can help prevent sexual problems. In addition, pre-marital sexual health education help females abandon self-containment and sexual inhibition and enjoy this natural desire for marital relation. Conversely, inadequate knowledge or inaccurate information can increase the vulnerability and dissatisfaction of couples. However, with regard to Iranian culture and religion, access to appropriate information leads to the improvement of sexual knowledge and higher sexual satisfaction. Therefore, the authors suggest that this counselling performance for all couples before marriage.

\section{Authors' Contribution}

All authors contributed to design of the work, data collection, data analysis, drafting this article and revision of the article.

\section{Conflict of Interests}

Authors declare that they have no conflict of interests.

\section{Ethical Issues}

The study protocol was approved by the Ethics Committee of Zahedan University of Medical Sciences, Zahedan, Iran after the confirmation of the study procedure (Ethics No. R.ZAUMS. REC.1397.006) with the RCT identifier of IRCT20171108037313N6 (https://www.irct.ir/trial/30650). In line with the research ethics principles, the objectives of the research were explained, and written informed consent was obtained from the participants before the study.

\section{Financial Support}

None.

\section{Acknowledgments}

This article was extracted from an MA thesis in counseling in midwifery. Hereby, the authors extend their gratitude to all participants for their contribution to conduct the study.

\section{References}

1. Dogan T, Tugut N, Golbasi Z. The relationship between sexual quality of life, happiness, and satisfaction with life in married Turkish women. Sex Disabil. 2013;31(3):239-247. doi:10.1007/s11195-013-9302-z

2. Masoumi SZ, Kazemi F, Nejati B, Parsa P, Karami M. Effect of sexual counseling on marital satisfaction of pregnant women referring to health centers in Malayer (Iran): an educational randomized experimental study. Electron Physician. 2017;9(1):3598-3604. doi:10.19082/3598

3. Mirghaforvand M, Alizadeh S, Asghari M, Tavananezhad $\mathrm{N}$, Karkhaneh $\mathrm{M}$. Individual-social predictor of marital satisfaction in women of reproductive age in Tabriz [Persian].
Iran J Obstet Gynecol Infertil. 2013;16(72):1-12.

4. Pakgohar M, Vizheh M, Babaee G, Ramezanzadeh F, Abedininia N. Effect of counseling on sexual satisfaction among infertile women referred to Tehran fertility center [Persian]. Hayat. 2008;14(1):21-30.

5. Rahmani A, Sadeghi N, Allahgholi L, Merghati-Khoei E. The relation of sexual satisfaction and demographic factors. Iran Journal of Nursing. 2010;23(66):14-22.

6. Sharyati M, Ghamarani A, Solati Dehkord SK, Abbasi Molid $\mathrm{H}$. The study of relationship between alexithymia and sexual satisfaction among female married students in Tabriz University [Persian]. J Fam Res. 2010;6(1):59-70.

7. Goldman JD. An exploration in health education of an integrated theoretical basis for sexuality education pedagogies for young people. Health Educ Res. 2011;26(3):526-541. doi:10.1093/her/cyq084

8. Latif Nejad R, Javad Nouri M, Hasanpour M, Hazaveyi SMM, Taghipour A. The necessity of sexual-health education for Iranian female adolescents: a qualitative study. Iran J Obstet Gynecol Infertil. 2012;15(12):7-17. doi:10.22038/ ijogi.2012.5695

9. World Health Organization (WHO. Medical Eligibility Criteria for Contraceptive Use. WHO; 2010.

10. Vaziri SH, Lotfi Kashani F, Hosseinian S, Bahram Ghafari S. Sexual efficacy and marital satisfaction [Persian]. Andisheh va Raftar. 2010;4(16):75-81.

11. Alimohammadi L, Zarei F, Mirghafourvand M. Factors associated with sexual dysfunction in newly married women referred to the urban health centers of Zanjan, Iran: a crosssectional study. Int J Womens Health Reprod Sci. 2018; 6(4):477-482. doi:10.15296/ijwhr.2018.79

12. Serap Kurbanoglu S, Akkoyunlu B, Umay A. Developing the information literacy self-efficacy scale. J Doc. 2006;62(6):730743. doi:10.1108/00220410610714949

13. Bandura A. Guide for constructing self-efficacy scales. 2006;5(1):307-337.

14. Kaviani M, Rahnavard T, Azima S, Emamghoreishi M, Asadi $N$, Sayadi M. The effect of education on sexual health of women with hypoactive sexual desire disorder: a randomized controlled trial. Int J Community Based Nurs Midwifery. 2014;2(2):94-102.

15. Farnam F, Pakgohar M, Mir-mohammadali M. Effect of pre-marriage counseling on marital satisfaction of Iranian newlywed couples: a randomized controlled trial. Sex Cult. 2011;15(2):141-152. doi:10.1007/s12119-010-9086-6

16. Parhizgar O, Esmaelzadeh-Saeieh S, Akbari Kamrani M, Rahimzadeh M, Tehranizadeh M. Effect of premarital counseling on marital satisfaction. Shiraz E-Med J. 2017;18(5):e13182. doi:10.5812/semj.44693

17. Stahmann RF. Premarital counselling: a focus for family therapy. J Fam Ther. 2000;22(1):104-116. doi:10.1111/14676427.00140

18. Bostani Khalesi Z, Simbar M. Challenges of premarital education program in Iran. J Holist Nurs Midwifery. 2017; 27(1):9-18. doi:10.18869/acadpub.hnmj.27.1.9

19. Mohebbi P, Malekpour P, Kamalifard M, Atri SB, Safaeiyan A, Alizadeh K. Clients' viewpoints about the quality of services in the premarital counseling classes in Tabriz health centers. Int J Prev Med. 2014;5(3):365-369.

20. Bostani Khalesi Z, Simbar M, Azin SA, Zayeri F. Public sexual health promotion interventions and strategies: $A$ qualitative study. Electron Physician. 2016;8(6):2489-2496. doi:10.19082/2489

21. Rosen R, Brown C, Heiman J, et al. The Female Sexual Function Index (FSFI): a multidimensional self-report instrument for 
the assessment of female sexual function. J Sex Marital Ther. 2000;26(2):191-208. doi:10.1080/009262300278597

22. Fakhri A, Pakpour AH, Burri A, Morshedi H, Mohammadi Zeidi I. The Female Sexual Function Index: translation and validation of an Iranian version. J Sex Med. 2012;9(2):514523. doi:10.1111/j.1743-6109.2011.02553.x

23. Alimohammadi L, Mirghafourvand M, Zarei F, Pirzeh R. The effectiveness of group counseling based on Bandura's selfefficacy theory on sexual function and sexual satisfaction in Iranian newlywed women: a randomized controlled trial. Appl Nurs Res. 2018;42:62-69. doi:10.1016/j.apnr.2018.06.011

24. Behboodi Moghadam Z, Rezaei E, Khaleghi Yalegonbadi F, et al. The effect of sexual health education program on women sexual function in Iran. J Res Health Sci. 2015;15(2):124-128.

25. Shams Mofaraheh Z, Botlani Esfahani S, Shahsiah M. The effect of marital counselling on sexual satisfaction of couples. J Hum Health. 2015;1(3):85-89.

26. Vural BK, Temel AB. Effectiveness of premarital sexual counselling program on sexual satisfaction of recently married couples. Sex Health. 2009;6(3):222-232. doi:10.1071/ sh08065

27. Byers ES. Relationship satisfaction and sexual satisfaction: a longitudinal study of individuals in long-term relationships. J Sex Res. 2005:42(2):113-118. doi:10.1080/00224490509552264

28. Sprecher S. Sexual satisfaction in premarital relationships: associations with satisfaction, love, commitment, and stability. J Sex Res. 2002;39(3):190-196. doi:10.1080/00224490209552141

29. TrudelG.Sexualityandmaritallife:resultsofasurvey.JSexMarital Ther.2002;28:229-249.doi:10.1080/009262302760328271

30. Reissing ED, Laliberté GM, Davis HJ. Young women's sexual adjustment: the role of sexual self-schema, sexual self- efficacy, sexual aversion and body attitudes. Can J Hum Sex. 2005; 14(3-4):77-85.

31. McCool ME, Zuelke A, Theurich MA, Knuettel H, Ricci C, Apfelbacher C. Prevalence of female sexual dysfunction among premenopausal women: a systematic review and metaanalysis of observational studies. Sex Med Rev. 2016;4(3):197212. doi:10.1016/j.sxmr.2016.03.002

32. McCool-Myers $M$, Theurich $M$, Zuelke A, Knuettel $H$, Apfelbacher C. Predictors of female sexual dysfunction: a systematic review and qualitative analysis through gender inequality paradigms. BMC Womens Health. 2018;18(1):108. doi:10.1186/s12905-018-0602-4

33. Meston CM, Hull E, Levin RJ, Sipski M. Disorders of orgasm in women. J Sex Med. 2004;1(1):66-68. doi:10.1111/j.17436109.2004.10110.x

34. Meston CM, Levin RJ, Sipski ML, Hull EM, Heiman JR. Women's orgasm. Annu Rev Sex Res. 2004;15:173-257.

35. Simbar M, Alizadeh S, Hajifoghaha M, Golezar S. Review of Iranian adolescents'educational needs for sexual and reproductive health. Journal of Isfahan Medical School. 2017;34(412):1563-1572. [Persian].

36. Dillon LM, Beechler MP. Marital satisfaction and the impact of children in collectivist cultures: a meta-analysis. J Evol Psychol. 2010;8(1):7-22. doi:10.1556/jep.8.2010.1.3

37. Zimmer-Gembeck MJ. Young females' sexual self-efficacy: associations with personal autonomy and the couple relationship. Sex Health. 2013;10(3):204-210. doi:10.1071/ sh12139

38. Karimi A, Dadgar S, Afiat M, Rahimi N. The effect of sexual health education on couples' sexual satisfaction. Iran J Obstet Gynecol Infertil. 2013;15(42):22-30. [Persian].

(c) 2021 The Author(s); This is an open-access article distributed under the terms of the Creative Commons Attribution License (http:// creativecommons.org/licenses/by/4.0), which permits unrestricted use, distribution, and reproduction in any medium, provided the original work is properly cited. 